Pacific Journal of Mathematic 


\section{ON A CERTAIN GENERALIZATION OF $\iota_{p}$ SPACES}

\section{JEANNE LADUKE}

An $\mathscr{E}_{p}$ space is a product of finite-dimensional $c_{p}$ spaces with a weighted $\ell_{p}$ norm on the product. The first theorem of this paper yields an isometric embedding of $\mathscr{E}_{p}$ into an appropriate $c_{p}$ space. From this theorem, known results about $c_{p}$ are used to deduce, among other things, the Clarkson inequalities for $\mathscr{E}_{p}, 1<p<\infty$, and hence, the uniform convexity of $\mathscr{E}_{p}$ for $1<p<\infty$.

The second theorem characterizes the conjugate space of $\mathscr{E}_{p}$ for $0<p<1$. This result is then used to describe some spaces of multipliers. Let $\mathscr{A}$ and $\mathscr{B}$ be $\mathscr{E}_{p}$ spaces, $1 \leqq$ $p \leqq \infty$, or $\mathscr{E}_{0}$. The spaces $\mathscr{C}(\mathscr{A}, \mathscr{B})$ of multipliers from $\mathscr{A}$ to $\mathscr{B}$ have previously been identified with certain subspaces of $\mathscr{E}(I)$ and determined precisely in some cases. The third theorem is a complete description of these multiplier spaces: the cases $0<p<1$ are included and the spaces $\mathscr{L}(\mathscr{A}, \mathscr{B})$ are determined precisely for all pairs $\mathscr{A}, \mathscr{B}$.

1. Definitions. First, we repeat the definition of $c_{p}$ (called $C_{p}$ by Dunford and Schwartz [1], $S_{p}$ by Gohberg and Krein [2], and $c_{p}$ by McCarthy [6]). See also [3, D. 37] for the case where $H$ is finitedimensional.

Definition 1.1. Let $H$ be a Hilbert space and let $X$ be a compact operator on $H$. Then $X X^{*}$ is positive and compact and hence has a unique positive square root which is also compact. We denote this square root by $|X|$. Now let $\mu_{n}$ be the, at most countably many, nonzero eigenvalues of $|X|$ enumerated with their multiplicity and arranged in a decreasing sequence as $\mu_{1} \geqq \mu_{2} \geqq \cdots \geqq 0$. For $0<$ $p<\infty$, we define

$$
\|X\|_{\phi_{p}}=\left(\sum_{n=1}^{\infty} \mu_{n}^{p}\right)^{1 / p}
$$

whether finite or infinite; and we define

$$
\|X\|_{\phi_{\infty}}=\sup \left\{\mu_{n}: 1 \leqq n<\infty\right\}=\mu_{1} \text {. }
$$

Equivalently, [1, p. 1089], $\|X\|_{\dot{\phi}_{\infty}}$ is the operator norm of $X$. Then $c_{p}$ consists of all compact $X$ with $\|X\|_{\phi_{p}}$ finite.

See [1], [2], and [6] for a detailed treatment of $c_{p}$ spaces and for additional references. Also, [3, Appendix D] contains a number of results in case $H$ is finite-dimensional. 
We proceed to define $\mathscr{E}_{p}$ spaces. These spaces were introduced by R. A. Kunze [5] primarily for the purpose of having analogues of $\iota_{p}$ spaces in the study of harmonic analysis on compact non-Abelian groups. They have been studied and exploited for this purpose especially by Hewitt and Ross [3].

Definition 1.2. Let $I$ be an index set. For each $\iota \in I$, let $H_{c}$ be a finite-dimensional Hilbert space and let $a_{\iota} \geqq 1$. We let $\mathscr{E}(I)$ denote the $*$-algebra $\prod_{\llcorner\in I} \mathscr{\mathscr { B }}\left(H_{\iota}\right)$ with all operations defined coordinatewise. Let $E=\left(E_{\iota}\right)_{\iota \in I} \in \mathscr{E}(I)$. For $0<p<\infty$, we define

$$
\|E\|_{p}=\left(\sum_{\imath \in I} a_{\imath}\left\|E_{\imath}\right\|_{\rho_{p}}\right)^{1 / p}
$$

we also define

$$
\|E\|_{\infty}=\sup \left\{\left\|E_{\iota}\right\|_{i_{\infty}}: \iota \in I\right\} \text {. }
$$

For $0<p \leqq \infty, \mathscr{E}_{p}(I)$ is defined to be the set of all $E \in \mathscr{E}(I)$ for which $\|E\|_{p}$ is finite. In addition, $\mathscr{E}_{00}(I)$ is the set of $E \in \mathscr{E}(I)$ for which $\left\{\iota \in I: E_{\iota} \neq 0\right\}$ is finite; and $\mathscr{E}_{0}(I)$ is the set of $E \in \mathscr{E}(I)$ for which $\left\{\iota \in I:\left\|E_{\iota}\right\|_{\phi_{\infty}} \geqq \varepsilon\right\}$ is finite for all $\varepsilon>0$. Frequently we write $\mathscr{E}_{p}$ in place of $\mathscr{E}_{p}(I)$. We notice that if each $H_{\iota}$ is one-dimensional, then $\mathscr{C}_{p}(I)$ is just the $\left\{a_{\imath}\right\}$-weighted $\iota_{p}$ space which we will call $L_{p}$; namely, $\left\{c_{\iota}\right\}_{\iota \in I} \in L_{p}$ if and only if $c_{\iota} \in K$ for each $\iota \in I$ and $\|c\|_{p}=\left(\sum_{\iota \in I} a_{\iota}\left|c_{\iota}\right|^{p}\right)^{1 / p}<$ $\infty$. In addition, if each $a_{\iota}=1$, then $\mathscr{E}_{p}(I)$ is just $\iota_{p}(I)$. Also, it is convenient to think of $\mathscr{E}_{p}$ as a product of $c_{p}$ spaces with a weighted $\iota_{p}$ norm on the product.

2. An embedding theorem and some consequences. In Hewitt and Ross [3], several basic facts about $\mathscr{E}_{p}$ for $1 \leqq p \leqq \infty$ are proved. There it is shown that Hölder's inequality, Minkowski's inequality and certain generalizations of these hold. The major result of this section is (2.2), a theorem describing a linear isometry of $\mathscr{E}_{p}$ onto a subspace of an appropriate $c_{p}$ space. The theorem is then used to derive a number of inequalities for $\mathscr{E}_{p}$ from results known about $c_{p}$. We begin with a description of the setting.

Let $I$ be an index set and let $H_{c}$ be a finite-dimensional Hilbert space for each $\iota \in I$. Also, let $a_{\iota} \geqq 1$ for each $\iota \in I$. For $0<p \leqq \infty$, $\|E\|_{p}$ and $\mathscr{E}_{p}$ will be as in (1.2). Now from the Hilbert space direct sum $\bigoplus_{\imath \in I} H_{i}$; namely

$$
\bigoplus_{\imath \in I} H_{\iota}=\left\{\left\{\xi_{\iota}\right\} \in \prod_{\iota \in I} H_{\imath}: \sum_{i \in I}\left\|\xi_{\iota}\right\|^{2}<\infty\right\}
$$

with addition and scalar multiplication defined coordinatewise and with 
an inner product defined by $\left\langle\left\{\xi_{\imath}\right\},\left\{\eta_{l}\right\}\right\rangle=\sum_{\iota \in I}\left\langle\xi_{l}, \eta_{l}\right\rangle$. It is well known that $\bigoplus_{\iota \in I} H_{\iota}$ is a Hilbert space under these definitions.

Definition 2.1. Let $0<p<\infty$ and let $E=\left(E_{\iota}\right)_{\iota \in I} \in \mathscr{E}_{p}$. Define $T_{p}(E)=T_{E}$ where $T_{E}\left(\left\{\xi_{\iota}\right\}\right)=\left\{a_{\iota}^{1 / p} E_{\iota}\left(\xi_{\iota}\right)\right\}$ for all $\left\{\xi_{\iota}\right\} \in \bigoplus_{\iota \in I} H_{\iota}$. If $p=\infty$ and $E \in \mathscr{E}_{\infty}$, let $T_{\infty}(E)=T_{E}$ where $T_{E}\left(\left\{\xi_{\iota}\right\}\right)=\left\{E_{\iota}\left(\xi_{\iota}\right)\right\}$.

If $p=\infty$, it is known that $T_{E} \in \mathscr{B}\left(\bigoplus_{\imath \in I} H_{\imath}\right)$ and $\left\|T_{E}\right\|=\|E\|_{\infty}$. In general we have the following theorem.

THEOREM 2.2. Let $0<p<\infty$ and let $T_{p}$ be defined as above. Then $T_{p}$ is a linear, *-preserving isometry of $\mathscr{E}_{p}(I)$ onto the subspace $e_{p}=\left\{T \in c_{p}\left(\bigoplus_{\iota \in I} H_{\iota}\right): H_{\imath}\right.$ is invariant under $T$ for all $\left.\iota \in I\right\}$ of $c_{p}\left(\Theta_{\iota \in I} H_{\iota}\right)$.

Proof. First, let $\xi=\left\{\xi_{\iota}\right\} \in \bigoplus_{\iota \in I} H_{\iota}$ so that $T_{E}\left(\left\{\xi_{\iota}\right\}\right)=\left\{a_{\iota}^{1 / p} E_{\iota} \xi_{\iota}\right\}$ for $E=\left(E_{\iota}\right)_{\llcorner\in I} \in \mathscr{E}_{p}$. Then using [1, p. 1093, 9 (a)] to obtain the second inequality below, we have

$$
\begin{aligned}
\left\|T_{E}\left(\left\{\xi_{\iota}\right\}\right)\right\|^{2} & =\sum_{\iota \in I}\left\|a_{\iota}^{1 / p} E_{\iota}\left(\xi_{\iota}\right)\right\|^{2} \\
& \leqq \sum_{\iota \in I} a_{\iota}^{2 / p}\left\|E_{\iota}\right\|_{\phi_{\infty}}^{2}\left\|\xi_{\iota}\right\|^{2} \\
& \leqq \sum_{\iota \in I} a_{\iota}^{2 / p}\left\|E_{\iota}\right\|_{\phi_{p}}^{2}\left\|\xi_{\iota}\right\|^{2} \\
& =\sum_{\iota \in I}\left(a_{\iota}\left\|E_{\iota}\right\| \|_{\phi_{p}}^{p}\right)^{2 / p}\left\|\xi_{\iota}\right\|^{2} \\
& \leqq \sum_{\iota \in I}\|E\|_{p}^{2}\left\|\xi_{\iota}\right\|^{2} \\
& =\|E\|_{p}^{2}\|\xi\|^{2} .
\end{aligned}
$$

Therefore, $T_{E}\left(\left\{\xi_{\iota}\right\}\right) \in \bigoplus_{\iota \in I} H_{\iota}$ and $\left\|T_{E}\left(\left\{\xi_{\imath}\right\}\right)\right\| \leqq\|E\|_{p}\|\xi\|$. Also, $T_{E}$ is clearly linear. Hence, $T_{E} \in \mathscr{B}\left(\bigoplus_{\iota \in I} H_{\iota}\right)$ and $\left\|T_{E}\right\| \leqq\|E\|_{p}$. It is easy to check that $T_{p}$ is linear and $*$-preserving.

We must now see that $T_{E}$ is compact for $E \in \mathscr{E}_{p}$. Since $E \rightarrow T_{E}$ is continuous and $\mathscr{E}_{00}$ is dense in $\mathscr{E}_{p}$, we need only note that $T_{E}$ is compact for $E \in \mathscr{E}_{00}$. This is obvious since $T_{E}$ has finite-dimensional range for $E \in \mathscr{E}_{00}$.

To see that $T_{p}$ is an isometry, we make the following observation. Suppose $\left\{\phi_{\lambda}^{j}: j=1,2, \cdots, d_{\lambda}\right\}$ is an orthonormal basis for $H_{2}$ of dimension $d_{\lambda}$ for each $\lambda \in I$. For each $\lambda \in I$ and $j=1,2, \cdots, d_{\lambda}$, let $\phi^{\lambda, j}=$ $\left(\phi_{\iota}^{\lambda, j}\right)_{\iota \in I} \in \bigoplus_{\iota \in I} H_{\iota}$ be defined by

$$
\phi_{\iota}^{\lambda, j}= \begin{cases}\phi_{\lambda}^{j} & \text { if } \iota=\lambda \\ 0 & \text { if } \iota \neq \lambda .\end{cases}
$$

Then it is easy to see that $\left\{\dot{\phi}^{\lambda, j}: \lambda \in I, j=1,2, \cdots, d_{\lambda}\right\}$ is an orthonormal basis for $\bigoplus_{\imath \in I} H_{\imath}$. Now, let $E \in \mathscr{E}_{p}$ and let $\left\{\beta_{\lambda}^{(j)}: j=1,2, \cdots, d_{\lambda}\right\}$ be 
the eigenvalues of $\left|E_{\lambda}\right|$ for each $\lambda \in I$. For each $\lambda \in I$, we choose $\left\{\phi_{\lambda}^{j}: j=1,2, \cdots, d_{\lambda}\right\}$ to be an orthonormal basis for $H_{\lambda}$ consisting of eigenvectors corresponding to the eigenvalues $\left(\beta_{\lambda}^{(j)}\right)^{2}$ of $E_{\lambda} E_{\lambda}^{*}$; that is, $E_{\lambda} E_{\lambda}^{*} \phi_{\lambda}^{j}=\left(\beta_{\lambda}^{(j)}\right)^{2} \phi_{\lambda}^{j}$. Letting $\phi^{\lambda, j}$ be as above, we have that $T_{E} T_{E}^{*} \phi^{\lambda, j}=$ $T_{E} T_{E} * \phi^{\lambda, j}=\left\{\eta_{\imath}\right\}_{\llcorner\in I}$ where $\eta_{\imath}=a_{\lambda}^{2 / p} E_{\lambda} E_{\lambda}^{*} \phi_{\lambda}^{j}=a_{\lambda}^{2 / p}\left(\beta_{\lambda}^{(j)}\right)^{2} \phi_{\lambda}^{j}$, if $\iota=\lambda$ and $\eta_{\imath}=0$ for $\iota \neq \lambda$. That is, $T_{E} T_{E}^{*} \phi^{\lambda, j}=\left(\alpha_{\lambda}^{1 / p} \beta_{\lambda}^{(j)}\right)^{2} \phi^{\lambda, j}$; or $\left\{\phi^{\lambda, j}: \lambda \in I, j=\right.$ $\left.1, \cdots, d_{\lambda}\right\}$ is an orthonormal basis for $\bigoplus_{\iota \in I} H_{\iota}$ consisting of eigenvectors corresponding to the eigenvalues $\left(a_{\lambda}^{1 / p} \beta_{\lambda}^{(j)}\right)^{2}$ of $T_{E} T_{E}^{*}$. Hence, by definition, we have

$$
\begin{aligned}
\left\|T_{E}\right\|_{p}^{p} & =\sum_{\substack{i \in I \\
j=1,2, \cdots, d_{\lambda}}}\left(a_{\lambda}^{1 / p} \beta_{\lambda}^{(j)}\right)^{p} \\
& =\sum_{\lambda \in I} a_{\lambda} \sum_{j=1}^{d_{\lambda}}\left(\beta_{\lambda}^{(j)}\right)^{p} \\
& =\sum_{\lambda \in I} a_{\lambda}\left\|E_{\lambda}\right\|_{\phi_{p}}^{p}=\|E\|_{p}^{p} .
\end{aligned}
$$

Thus, $T_{p}$ is an isometry.

Finally, we show that $T_{p}$ maps $\mathscr{E}_{p}$ onto $e_{p}\left(\bigoplus_{\iota \in I} H_{\iota}\right)$. Consider $S$ in $e_{p}\left(\bigoplus_{\iota \in I} H_{\iota}\right)$. For each $\iota \in I$, we let $E_{\iota}=\left.a_{\iota}^{-1 / p} S\right|_{H_{\iota}}$. Since $H_{\imath}$ is invariant under $S, E_{\iota} \in \mathscr{B}\left(H_{\imath}\right)$ for each $\iota \in I$. Also, we notice that $H_{\imath}$ is invariant under $S^{*}$ for each $\iota \in I$. Hence, for $\xi_{l}, \eta_{\iota} \in H_{\iota}$, we have

$$
\begin{aligned}
\left\langle E_{\imath} \xi_{\iota}, \eta_{\imath}\right\rangle & =\left\langle\left. a_{t}^{-1 / p} S\right|_{H_{\iota}} \xi_{\iota}, \eta_{\imath}\right\rangle \\
& =a_{\imath}^{-1 / p}\left\langle\xi_{\iota},\left.S^{*}\right|_{H_{\iota}} \eta_{\imath}\right\rangle \\
& =\left\langle\xi_{\iota},\left.a_{\iota}^{-1 / p} S^{*}\right|_{H_{\iota}} \eta_{\imath}\right\rangle
\end{aligned}
$$

and so $E_{\iota}^{*}=\left.a_{\iota}^{-1 / p} S^{*}\right|_{H_{\iota}}$ for each $\iota \in I$. Now we essentially repeat an earlier argument. Namely, let $\left\{\beta_{\lambda}^{(j)}: j=1,2, \cdots, d_{\lambda}\right\}$ be eigenvalues of $\left|E_{\lambda}\right|$ for each $\lambda \in I$ and let $\left\{\phi_{\lambda}^{j}: j=1, \cdots, d_{\lambda}\right\}$ be an orthonormal basis for $H_{\lambda}$ consisting of eigenvectors corresponding to the eigenvalues $\left\{\left(\beta_{\lambda}^{(j)}\right)^{2}: j=1, \cdots, d_{\lambda}\right\}$ of $E_{\lambda} E_{\lambda}^{*}$. Then, as above, $S S^{*} \phi^{\lambda, j}=a_{\lambda}^{2 / p}\left(\beta_{\lambda}^{(j)}\right)^{2} \phi^{\lambda, j}$ so that $\|S\|_{p}^{p}=\|E\|_{p}^{p}$ where $E=\left(E_{\iota}\right)_{\iota \in I}$, and hence $E \in \mathscr{E}_{p}$. Clearly, $S(\xi)=T_{E}(\xi)$ for all $\xi \in H_{\iota}, \iota \in I$; thus, by linearity, $S(\xi)=T_{E}(\xi)$ for all $\xi \in \bigoplus_{\iota \in I} H_{\imath}$ with $\xi_{\iota} \neq 0$ for only finitely many $\iota \in I$. By the density of the latter set in $\bigoplus_{\iota \in I} H_{\iota}, S(\xi)=T_{E}(\xi)$ for all $\xi \in \bigoplus_{\iota \in I} H_{\iota}$. Hence $T_{p}(E)=S$ and so $T_{p}$ maps onto $e_{p}\left(\bigoplus_{\iota \in I} H_{\iota}\right)$.

We state several corollaries which follow immediately from results for $c_{p}$ spaces found in [1, XI, §9], [2, III, §7] and [6]. Also, compare $[3, \S 28]$.

CoRollary 2.3. Let $0<p \leqq q \leqq \infty$. Then $\mathscr{E}_{p}(I) \subset \mathscr{E}_{q}(I)$ and $\|E\|_{q} \leqq\|E\|_{p}$.

Corollary 2.4. Suppose $0<p \leqq 1$; let $E, F \in \mathscr{E}_{p}(I)$. Then

$$
\|E+F\|_{p}^{p} \leqq\|E\|_{p}^{p}+\|F\|_{p}^{p} .
$$


Thus, $\mathscr{C}_{p}(I)$ is a metric space with metric $\rho$ where $\rho(A, B)=\|A-B\|_{p}^{p}$.

Inequalities (i) and (ii) in the following are due to McCarthy [6, Th. 2.7] for $c_{p}$ spaces.

CoROllaRY 2.5. (Clarkson's inequalities). Let $E, F \in \mathscr{E}(I)$. Then, for $1 / p+1 / p^{\prime}=1$, we have

( i ) $2^{p-1}\left(\|E\|_{p}^{p}+\|F\|_{p}^{p}\right) \leqq\|E+F\|_{p}^{p}+\|E-F\|_{p}^{p} \leqq 2\left(\|E\|_{p}^{p}+\|F\|_{p}^{p}\right)$ $0<p \leqq 2$,

(ii) $\|E+F\|_{p}^{p^{\prime}}+\|E-F\|_{p}^{p^{\prime}} \leqq 2\left(\|E\|_{p}^{p}+\|F\|_{p}^{p}\right)^{p^{\prime} / p} 1<p \leqq 2$,

(iii) $2\left(\|E\|_{p}^{p}+\|F\|_{p}^{p}\right) \leqq\|E+F\|_{p}^{p}+\|E-F\|_{p}^{p} \leqq 2^{p-1}\left(\|E\|_{p}^{p}+\|F\|_{p}^{p}\right)$ $2 ! \leqq p<\infty$,

(iv) $2\left(\|E\|_{p}^{p}+\|F\|_{p}^{p}\right)^{p^{\prime} / p} \leqq\|E+F\|_{p}^{p^{\prime}}+\|E-F\|_{p}^{p^{\prime}} 2 \leqq p<\infty$.

CoRollary 2.6. For $1<p<\infty, \mathscr{E}_{p}(I)$ is uniformly convex. (Recall that a normed linear space $X$ is said to be uniformly convex if $\delta(\varepsilon)=\inf \{1-1 / 2|x+y|:|x|=|y|=1,|x-y|=\varepsilon\}$ is strictly positive in some range $0<\varepsilon<\varepsilon_{0}$.)

Proof. Use the Clarkson inequalities (2.5) (ii) and the right hand half of (2.5) (iii) to obtain

$$
\|E+F\|_{p}^{p^{\prime}} \leqq 2^{p^{\prime}}-\|E-F\|_{p}^{p^{\prime}} \text { for } 1<p \leqq 2
$$

and

$$
\|E+F\|_{p}^{p} \leqq 2^{p}-\|E-F\|_{p}^{p} \text { for } 2 \leqq p<\infty
$$

when $\|E\|_{p}=\|F\|_{p}=1$. If, in addition, $\|E-F\|_{p}=\varepsilon$, we have

$$
1-\frac{1}{2}\|E+F\|_{p} \geqq 1-\frac{1}{2}\left(2^{p^{\prime}}-\varepsilon^{p^{\prime}}\right)^{1 / p^{\prime}} \text { for } 1<p \leqq 2 ，
$$

and

$$
1-\frac{1}{2}\|E+F\|_{p} \geqq 1-\frac{1}{2}\left(2^{p}-\varepsilon^{p}\right)^{1 / p} \text { for } 2 \leqq p<\infty .
$$

The uniform convexity of $\mathscr{E}_{p}$ for $1<p<\infty$ is now clear.

CoRollary 2.7. (Radon-Riesz theorem). Let $1<p<\infty$. Let $\left(E^{(n)}\right)$ be a sequence in $\mathscr{E}_{p}(I)$ and $E \in \mathscr{E}_{p}(I)$ such that $E^{(n)} \rightarrow E$ weakly and $\left\|E^{(n)}\right\|_{p} \rightarrow\|E\|_{p}$. Then $\left\|E^{(n)}-E\right\|_{p} \rightarrow 0$.

Proof. $\mathscr{E}_{p}(I)$ is locally uniformly convex; see $[4,15.17$ (a)]. Hence, apply [4, 15.17 (a)]. 
3. The conjugate space of $\mathscr{E}_{p}$ for $0<p<1$. Theorem (3.4) below is a characterization of the conjugate space of $\mathscr{E}_{p}$ for $0<p<1$. The conjugate spaces of $\mathscr{E}_{p}$ for $1 \leqq p<\infty$ are described in [3, §28]. We first state and prove some easy results which will be used in the proof of (3.4).

Lemma 3.1. Let $H$ be a finite-dimensional Hilbert space and let $0<p, q \leqq \infty$. For each $A \in \mathscr{B}(H)$, there exists $B \in \mathscr{B}(H)$ such that $\|B\|_{\phi_{p}}=1$ and $\|A\|_{\phi_{\infty}}=\|A B\|_{\phi_{q}}=\operatorname{tr}(A B)$.

Proof. (Compare [3, D.54].) Let $a$ be the eigenvalue of $|A|$ such that $a=\|A\|_{\phi_{\infty}}$. By [3, D.30] there is an operator $V$ in $\mathscr{U}(H)$ such that $A V=|A|$. Let $\left\{\zeta_{1}, \zeta_{2}, \cdots, \zeta_{n}\right\}$ be a basis for $H$ such that $|A| \zeta_{1}=$ $a \zeta_{1}$. Let $P$ be the operator on $H$ such that $P \zeta_{1}=\zeta_{1}$ and $P \zeta_{j}=0$ for $j>1$. Finally, let $B=V P$. By $\left[1, \mathrm{p} .1090,4\right.$ (c)], we have $\|B\|_{\phi_{p}}=$ $\|P\|_{\phi_{p}}=1$. Since $A B=A V P=|A| P$, we have $A B=\alpha P$, and hence

$$
\|A B\|_{\phi_{q}}=\|a P\|_{\phi_{q}}=a=\|A\|_{\phi_{\infty}},
$$

and

$$
\operatorname{tr}(A B)=\operatorname{tr}(a P)=a=\|A\|_{\phi_{\infty}} .
$$

LEMMA 3.2. Let $H$ be a finite-dimensional Hilbert space, and let $A \in \mathscr{B}(H)$. Then

(i) For $0<p \leqq q \leqq \infty$, we have

$$
\|A\|_{\phi_{\infty}}=\sup \left\{\|A B\|_{\phi_{q}}: B \in \mathscr{B}(H) \text { and }\|B\|_{\phi_{p}} \leqq 1\right\} \text {, }
$$

and

(ii) for $0<p \leqq 1$, we have

$$
\|A\|_{\phi_{\infty}}=\sup \left\{|\operatorname{tr}(A B)|: B \in \mathscr{B}(H) \text { and }\|B\|_{\phi_{p}} \leqq 1\right\} \text {. }
$$

Also, the supremum is attained in (i) and (ii).

Proof. Let $\alpha=\sup \left\{\|A B\|_{\phi_{q}}:\|B\|_{\phi_{p}} \leqq 1\right\}$ for $0<p \leqq q \leqq \infty$. Then by $[1$, p. 1093,9 (d) and 9 (a)],

$$
\|A B\|_{\phi_{q}} \leqq\|A\|_{\phi_{\infty}}\|B\|_{\phi_{q}} \leqq\|A\|_{\phi_{\infty}}\|B\|_{\phi_{p}} \leqq\|A\|_{\phi_{\infty}},
$$

so that $\alpha \leqq\|A\|_{\phi_{\infty}}$.

For $0<p \leqq 1$, let $\beta=\sup \left\{|\operatorname{tr}(A B)|:\|B\|_{\phi_{p}} \leqq 1\right\}$. By [3, D.46], we have

$$
|\operatorname{tr}(A B)| \leqq\|A B\|_{\phi_{1}} \leqq\|A\|_{\phi_{\infty}}\|B\|_{\phi_{1}} \leqq\|A\|_{\phi_{\infty}}\|B\|_{\phi_{p}} \leqq\|A\|_{\phi_{\infty}}
$$

so that $\beta \leqq\|A\|_{\phi_{\infty}}$. 
The opposite inequalities and the fact that the supremum is attained in (i) and (ii) follow from (3.1).

Lemma 3.3. Let $0<p<1, E \in \mathscr{E}_{p}(I)$ and $F \in \mathscr{E}_{\infty}(I)$. Then $E F$ and $F E$ are in $\mathscr{E}_{p}(I)$,

(i ) $\|E F\|_{p} \leqq\|E\|_{p}\|F\|_{\infty}$, and

(ii) $\|F E\|_{p} \leqq\|F\|_{\infty}\|E\|_{p}$.

Proof. Use [1, p. 1093, 9 (d)] to write

$$
\begin{aligned}
\|E F\|_{p}^{p} & =\sum_{\iota \in I} a_{\iota}\left\|E_{\iota} F_{\iota}\right\|_{\phi_{p}}^{p} \leqq \sum_{\iota \in I} a_{\iota}\left\|E_{\iota}\right\|_{\phi_{p}}^{p}\left\|F_{\iota}\right\|_{\phi_{\infty}}^{p} \\
& \leqq\|F\|_{\infty}^{p} \sum_{\iota \in I} a_{\iota}\left\|E_{\iota}\right\|_{\phi_{p}}^{p}=\|F\|_{\infty}^{p}\|E\|_{p}^{p} .
\end{aligned}
$$

Assertion (ii) follows similarly.

Theorem 3.4. Let $0<p<1$, and let $F \in \mathscr{E}(I)$. If there exists a real number $c>0$ such that $\left\|F_{\iota}\right\|_{\phi_{\infty}} \leqq c a_{\iota}^{(1 / p)-1}$ for all $\iota \in I$, then $T_{F}$, defined on $\mathscr{E}_{p}(I)$ by $T_{F}(E)=\langle E, F\rangle=\sum_{\iota \in I} a_{\iota} \operatorname{tr}\left(E_{\iota} F_{\iota}^{*}\right)$, is a continuous linear functional on $\mathscr{E}_{p}(I)$. Conversely, if $T$ is a continuous linear functional on $\mathscr{E}_{p}(I)$, then $T=T_{F}$ for some $F \in \mathscr{E}(I)$ such that $\left\|F_{\imath}\right\|_{\phi_{\infty}} \leqq c a^{(1 / p)-1}$ for some $c>0$ and all $\iota \in I$.

Proof. First, suppose there exists $c>0$ such that $\left\|F_{\iota}\right\|_{\phi_{\infty}} \leqq$ $c a_{\iota}^{(1 / p)-1}$ for all $\iota \in I$. Then, for $E \in \mathscr{E}_{p}(I)$, the number $T_{F}(E)=$ $\sum_{\iota \in I} a_{\iota} \operatorname{tr}\left(E_{\iota} F_{\iota}^{*}\right)$ is well-defined (the series converges absolutely) since by (3.2) and an observation below, we have

$$
\begin{aligned}
\mid T_{F}(E) & =\left|\sum_{\iota \in I} a_{\iota} \operatorname{tr}\left(E_{\iota} F_{\iota}^{*}\right)\right| \\
& \leqq \sum_{\iota \in I} a_{\iota}\left|\operatorname{tr}\left(E_{\iota} F_{\iota}^{*}\right)\right| \\
& \leqq \sum_{\iota \in I} a_{\iota}\left\|E_{\iota}\right\|_{\phi_{p}}\left\|F_{\iota}\right\|_{\phi_{\infty}} \\
& \leqq \sum_{\iota \in I} c a_{\iota}^{1 / p}\left\|E_{\iota}\right\|_{\phi_{p}} \\
& =c \sum_{\iota \in I}\left(a_{\iota}\left\|E_{\iota}\right\|_{\phi_{p}}^{p}\right)^{1 / p} \\
& \leqq c\left[\sum_{\iota \in I} a_{\iota}\left\|E_{\iota}\right\|_{\phi_{p}}^{p}\right]^{1 / p}=c\|E\|_{p} .
\end{aligned}
$$

The last inequality follows since $1<1 / p$ so that $\|b\|_{1 / p} \leqq\|b\|_{1}$ for $b \in \ell_{1}$, and in particular for $b=\{b\}$ where $b_{\iota}=a_{\iota}\left\|E_{\iota}\right\|_{\phi_{p}}^{p}$.

The linearity of $T_{F}$ follows immediately from the linearity of $\operatorname{tr}$ [3, D.16]. The inequality (1) also shows that $T_{F}$ is continuous at 0 , hence on $\mathscr{E}_{p}(I)$. (Recall that $\mathscr{E}_{p}(I)$ is a metric spaces with $\rho(A, B)=$ $\|A-B\|_{p}^{p}$.) Thus, $T_{F}$ is a continuous linear functional on $\mathscr{E}_{p}(I)$. 
Conversely, let $T$ be a continuous linear functional on $\mathscr{E}_{p}(I)$. Let $\mathscr{A}_{l}=\left\{E \in \mathscr{E}_{p}(I): E_{\lambda}=0\right.$ for $\left.\lambda \neq \iota\right\}$. Then $\mathscr{A}$ c is isomorphic with $\mathscr{B}\left(H_{6}\right)$. Restricting $T$ to $\mathscr{A}_{1}$, we use elementary algebra to see that there exists $F_{\iota} \in \mathscr{B}\left(H_{\imath}\right)$ such that $T(E)=a, \operatorname{tr}\left(E_{\iota} F_{\iota}^{*}\right)$, for all $E \in \mathscr{K}_{\iota}$. The linearity of $T$ shows that

$$
T(E)=\sum_{\iota \in I} a_{\iota} \operatorname{tr}\left(E_{\iota} F_{\iota}^{*}\right)
$$

for all $E \in \mathscr{E}_{00}(I)$. Let $F=\left(F_{\iota}\right)_{\epsilon \in I}$, so that $T=T_{F}$ on $\mathscr{E}_{00}(I)$.

Now suppose that for every real number $c>0$, there exists $\iota \in I$ such that $\left\|F_{c}\right\|_{\phi_{\infty}}>c \alpha_{\iota}^{(1 / p)-1}$. In particular, for $n \in\{1,2, \cdots\}$, let $\iota_{n} \in I$ be such that $\iota_{n} \neq \iota_{m}$ for $m \neq n$ and $\left\|F_{c_{n}}\right\|_{\phi_{\infty}}>n^{k} \alpha_{\iota_{n}}^{(1 / p)-1}$, where $k$ is a real number greater than zero and such that $2 /(1+k)<p$.

For each $n \in\{1,2, \cdots\}$, let $B_{\iota_{n}} \in \mathscr{B}\left(H_{\iota_{n}}\right)$ be such that $\left\|B_{\iota_{n}}\right\|_{\phi_{p}}=$ 1 and $\left\|F_{\iota_{n}}\right\|_{\phi_{\infty}}=\operatorname{tr}\left(F_{\iota_{n}} B_{\iota_{n}}\right)$ as in (3.1). Let $b_{n}=\left(a_{\iota_{n}} n^{2}\right)^{-1 / p}$ for each $n$, and define $E=\left(E_{c}\right)_{\iota \in I}$, where $E,=b_{n} B_{\iota_{n}}^{*}$ if $\iota=\iota_{n}$ for some $n$, and $E_{\iota}=0$ otherwise. Then

$$
\begin{aligned}
\|E\|_{p}^{p} & =\sum_{\iota \in I} a_{\iota}\left\|E_{\iota}\right\|_{\phi_{p}}^{p}=\sum_{n=1}^{\infty} a_{\iota_{n}}\left\|b_{n} B_{\iota_{n}}^{*}\right\|_{\phi_{p}}^{p} \\
& =\sum_{n=1}^{\infty} a_{\iota_{n}} b_{n}^{p}\left\|B_{\iota_{n}}\right\|_{\rho_{p}}^{p}=\sum_{n=1}^{\infty} a_{\iota_{n}}\left(a_{\iota_{n}} n^{2}\right)^{-1} \\
& =\sum_{n=1}^{\infty} n^{-2}<\infty
\end{aligned}
$$

so that $E \in \mathscr{E}_{p}(I)$.

For each positive integer $N$, define $E^{(N)}=\left(E_{c}^{(N)}\right)_{\iota \in I}$, where $E_{c}^{(N)}=$ $E_{\iota}$ if $\iota=\iota_{n}$ with $n \leqq N$, and $E_{c}^{(N)}=0$ otherwise. Then $E^{(N)} \in \mathscr{E}_{00}(I)$ and $\left\|E^{(N)}\right\|_{p}^{p} \leqq\|E\|_{p}^{p}$ for each $N$. However,

$$
\begin{aligned}
T\left(E^{(N)}\right)=T_{F}\left(E^{(N)}\right) & =\sum_{\iota \in I} a_{\iota} \operatorname{tr}\left(E_{\iota}^{(N)} F_{\iota}^{*}\right) \\
& =\sum_{n=1}^{N} a_{\iota_{n}} \operatorname{tr}\left(E_{\iota_{n}} F_{\iota_{n}}^{*}\right) \\
& =\sum_{n=1}^{N} a_{\iota_{n}} \operatorname{tr}\left(b_{n} B_{\iota_{n}}^{*} F_{\iota_{n}}^{*}\right) \\
& =\sum_{n=1}^{N} a_{\iota_{n}} b_{n} \operatorname{tr}\left(\left(F_{\iota_{n}} B_{\iota_{n}}\right)^{*}\right) \\
& =\sum_{n=1}^{N} a_{\iota_{n}} b_{n} \overline{\operatorname{tr}\left(F_{\iota_{n}} B_{\iota_{n}}\right)} \\
& =\sum_{n=1}^{N} a_{\iota_{n}}\left(a_{\iota_{n}} n^{2}\right)^{-1 / p}\left\|F_{\iota_{n}}\right\|_{\phi_{\infty}} \\
& >\sum_{n=1}^{N} a_{\iota_{n}}\left(a_{\iota_{n}} n^{2}\right)^{-1 / p} n^{k} a_{\iota_{n}}^{(1 / p)-1} \\
& =\sum_{n=1}^{N} n^{k-2 / p}>\sum_{n=1}^{N} 1 / n .
\end{aligned}
$$


A simple argument now shows that $T$ is discontinuous, a contradiction. Therefore, there exists $c>0$ so that $\left\|F_{c}\right\|_{\phi_{\infty}} \leqq c a_{\iota}^{(1 / p)-1}$ for all $\iota \in I$. Thus, $T_{F}$ and $T$ are continuous linear functionals on $\mathscr{E}_{p}(I)$ which agree on $\mathscr{E}_{00}(I)$, a dense subspace of $\mathscr{E}_{p}(I)$, so that $T=T_{F}$ on $\mathscr{E}_{p}(I)$.

Several easy corollaries follow and will be stated without proof. The notation is as in (3.4).

COROLLARY 3.5. If $0<p<1$ and if $\sup _{\iota \in I} a_{\iota}<\infty$, then $\mathscr{E}_{p}^{*}=$ $\left\{T_{F}: F \in \mathscr{E}_{\infty}\right\}$.

CoROLLARY 3.6. Let $0<p<1$ and let $L_{p}$ be a weighted $\ell_{p}$ space; say $\|b\|_{p}=\left(\sum_{\iota \in I} a_{\ell}\left|b_{\iota}\right|^{p}\right)^{1 / p}$ for $\left\{b_{\iota}\right\} \in L_{p}$. For $b=\left\{b_{\iota}\right\} \in L_{p}$ and $c=\left\{c_{\iota}\right\}$, let $T_{c}(b)=\sum_{\iota \in I} a_{t} b_{\iota} \bar{c}_{\iota}$. Then

$$
L_{p}^{*}=\left\{T_{c}:\left|c_{\iota}\right| \leqq k a_{\iota}^{(1 / p)-1} \text { for some } k>0 \text { and all } \iota \in I\right\} .
$$

CoRollary 3.7. If $0<p<1$, then $\ell_{p}^{*}=\left\{T_{c}\right.$ : $\left.c \in \ell_{\infty}\right\}$.

4. Some multiplier theorems. Theorem (4.2) is a collection of results concerning $\left(\mathscr{C}_{p}, \mathscr{E}_{q}\right)$-multipliers. We use the following definition: Let $\mathscr{A}$ and $\mathscr{B}$ be subsets of $\mathscr{E}(I)$. We say that $E$ in $\mathscr{E}(I)$ is an $(\mathscr{A}, \mathscr{B})$-multiplier if $E A \in \mathscr{B}$ for all $A \in \mathscr{A}$. The set of all $(\mathscr{A}, \mathscr{B})$-multipliers is denoted by $\mathscr{C}(\mathscr{A}, \mathscr{B})$.

Clearly, multipliers may be discussed in a context much wider than that of $\mathscr{E}_{p}$ spaces. For example, it is known that $\ell_{r}=\mathscr{M}\left(\ell_{q}, \ell_{p}\right)$ for $0<p<q<\infty$ with $1 / r=1 / p-1 / q$. Also, it is shown in McCarthy [6, Ths. 2.3 and 5.1] that $\mathscr{C}\left(c_{q}, c_{p}\right)=c_{r}$ for $p, q$ and $r$ as above.

In Hewitt and Ross [3, 35.4] $\mathscr{C}(\mathscr{A}, \mathscr{B})$ is described for any pair $(\mathscr{A}, \mathscr{B})$ chosen from the spaces $\mathscr{E}_{p}, \mathscr{E}_{q}, \mathscr{E}_{0}, \mathscr{E}_{\infty}$ with $1 \leqq p<$ $q<\infty$ with the following exceptions: if $\sup _{\iota \in I} a_{\iota}=\infty$, it is shown only that $\mathscr{M}(\mathscr{A}, \mathscr{B}) \supsetneqq \mathscr{E}_{\infty}$, where $\mathscr{A}=\mathscr{E}_{p}$ and $\mathscr{B}=\mathscr{E}_{q}$ or $\mathscr{B}=\mathscr{E}_{0}$ with $1 \leqq p<q<\infty$. Our theorem which follows extends the results of $[3,35.4]$ to all $p$ and $q$ with $0<p<q<\infty$. Also, it identifies $\mathscr{C}(\mathscr{A}, \mathscr{B})$ precisely in the exceptions mentioned above when $\sup _{\epsilon_{I} I} a_{t}=\infty$. The major tool used in the identification of $\mathscr{H}(\mathscr{A}, \mathscr{B})$ in the cases where $\sup _{\iota \in I} a_{t}=\infty$ is (3.4), our characterization of $\mathscr{E}_{p}{ }^{*}$ for $0<p<1$.

Before stating our theorem we note that the following lemma may easily be verified using [6, Th. 2.3] and the generalized Hölder inequality.

LEMma 4.1. Let $0<p, q, r<\infty$ with $1 / p+1 / q=1 / r$. If $E \in$ $\mathscr{E}_{p}(I), F \in \mathscr{E}_{q}(I)$, then $E F \in \mathscr{E}_{r}(I)$ and $\|E F\|_{r} \leqq\|E\|_{p}\|F\|_{q}$. 
TheOREM 4.2. Let $0<p<q<\infty$ and let $r$ be so that $1 / r=$ $1 / p-1 / q$. For each space $\mathscr{A}$ listed to the left of the matrix below and each space $\mathscr{B}$ listed above the matrix, the corresponding entry of the matrix is exactly $\mathscr{l l}(\mathscr{A}, \mathscr{B})$.

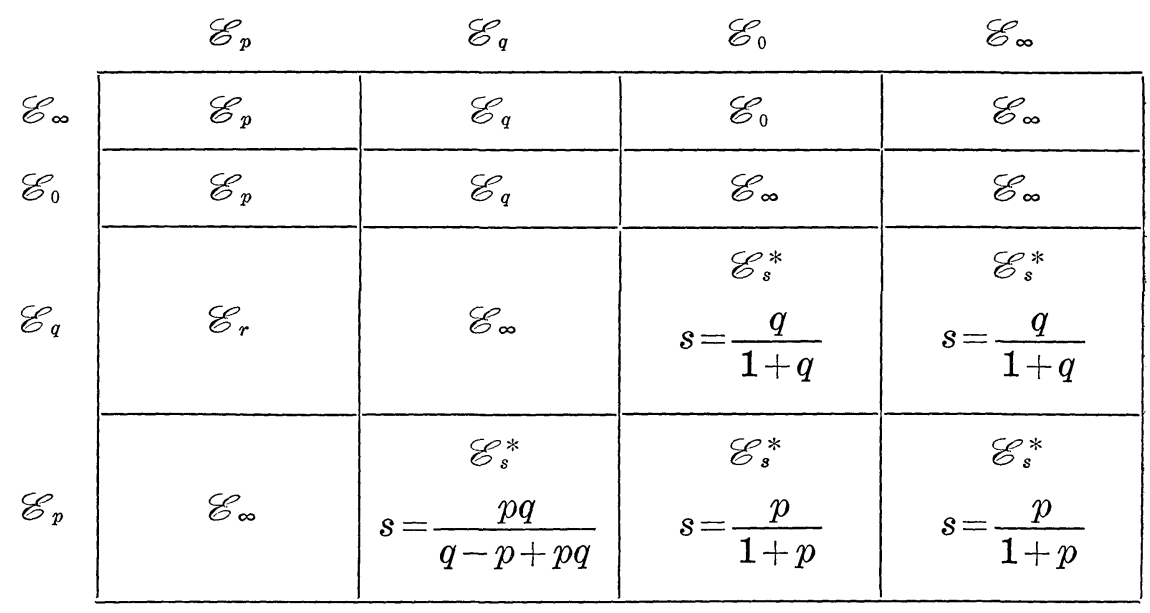

The proof of the above theorem will be broken into several parts.

Part I. For $0<p \leqq \infty, \mathscr{M}\left(\mathscr{E}_{p}, \mathscr{E}_{p}\right)=\mathscr{E}_{\infty}$.

Proof. In case $1 \leqq p \leqq \infty$, we use the proof of $[3,35.4$, Part II] with $d_{\sigma_{n}}$ replaced by $a_{\sigma_{n}}$ throughout.

Now let $0<p<1$. The fact that $\mathscr{E}_{\infty} \subset \mathscr{L}\left(\mathscr{E}_{p}, \mathscr{E}_{p}\right)$ follows from (3.3). The proof of the opposite inclusion is similar to the proof of [3, 35.4, Part II]. Namely, suppose $E \notin \mathscr{E}_{\infty}(I)$. Then there is a sequence $\left\{c_{n}\right\}_{n=1}^{\infty}$ of distinct elements in $I$ such that $\left\|E_{\iota_{n}}\right\|_{\dot{\phi}_{\infty}}>n$ for each $n$. By (3.1), there exists $B_{\iota_{n}}$ in $\mathscr{B}\left(H_{\iota_{n}}\right)$ such that $\left\|E_{\iota_{n}} B_{\iota_{n}}\right\|_{\phi_{p}}>n$ and $\left\|B_{\iota_{n}}\right\|_{\phi_{p}}=1$. For $n \in\{1,2, \cdots\}$, let $\alpha_{n}=\left(a_{\iota_{n}} n^{1+p}\right)^{-1 / p}$. Define $A \in$ $\mathscr{E}(I)$ as follows: $A_{\iota_{n}}=\alpha_{n} B_{\iota_{n}}$ for $n \in\{1,2, \cdots\}$ and $A_{\iota}=0$ for all other ¿'s in $I$. Since

$$
\begin{aligned}
\|A\|_{p}^{p} & =\sum_{n=1}^{\infty} a_{\iota_{n}}\left\|\alpha_{n} B_{\iota_{n}}\right\|_{\phi_{p}}^{p} \\
& =\sum_{n=1}^{\infty} n^{-(1+p)}<\infty,
\end{aligned}
$$

we have that $A \in \mathscr{E}_{p}(I)$. On the other hand, $E A$ does not belong to. $\mathscr{E}_{p}(I)$ because

$$
\begin{aligned}
\|E A\|_{p}^{p} & =\sum_{n=1}^{\infty} a_{\iota_{n}}\left\|\alpha_{n} E_{\iota_{n}} B_{\iota_{n}}\right\|_{\dot{\rho}_{p}}^{p} \geqq \sum_{n=1}^{\infty} a_{\iota_{n}}\left(a_{\iota_{n}} n^{1+p}\right)^{-1} n^{p} \\
& =\sum_{n=1}^{\infty} 1 / n=\infty .
\end{aligned}
$$


Thus, $E \notin \mathscr{M}\left(\mathscr{E}_{p}, \mathscr{E}_{p}\right)$ and so $\mathscr{M}\left(\mathscr{E}_{p}, \mathscr{E}_{p}\right) \subset \mathscr{E}_{\infty}(I)$. Hence, entries $(1,4),(3,2)$, and $(4,1)$ are verified.

Part II. For $0<p<\infty$, we have that $\mathscr{E}_{p}=\mathscr{M}\left(\mathscr{E}_{0}, \mathscr{E}_{p}\right)=$ $\mathscr{C}\left(\mathscr{E}_{\infty}, \mathscr{E}_{p}\right)$. This will verify entries $(1,1),(1,2),(2,1)$, and $(2,2)$.

Proof. Using (3.3) we see that, for $0<p<1, \mathscr{E}_{p} \subset \mathscr{M}\left(\mathscr{E}_{\infty}, \mathscr{E}_{p}\right) \subset$ $\mathscr{C}\left(\mathscr{E}_{0}, \mathscr{E}_{p}\right)$. The rest of the assertion is proved in [3, 35.4, Part VII] if we replace $d_{\sigma}$ by $a_{\sigma}$ throughout.

Part III. Let $0<p<q<\infty$ and let $s=p q /(q-p+p q)$. Then $\mathscr{E}_{s}^{*}=\mathscr{M}\left(\mathscr{E}_{p}, \mathscr{E}_{q}\right)$.

Proof. Consider $T_{F} \in \mathscr{E}_{s}^{*}$ with $s$ as above. Then $0<s<1$ so that by (3.4), there exists a real number $c>0$ such that $\left\|F_{c}\right\|_{\phi_{\infty}} \leqq$ $c a_{c}^{(1 / s)-1}$. Let $E \in \mathscr{E}_{p}$. The following is seen to be true by using \|\|$_{\iota_{q}} \leqq$ \|\|$_{\ell_{p}}$ for $0<p<q<\infty$ and the results (3.3), [3, D.52.i.], and (2.3).

$$
\begin{aligned}
\|F E\|_{q} & =\left[\sum_{\iota \in I}\left(a_{\iota}^{1 / q}\left\|F_{\iota} E_{\iota}\right\|_{\phi_{q}}\right)^{q}\right]^{1 / q} \\
& \leqq\left[\sum_{\iota \in I}\left(a_{\iota}^{1 / q}\left\|F_{\iota} E_{\iota}\right\|_{\phi_{q}}\right)^{p}\right]^{1 / p} \\
& \leqq\left[\sum_{\iota \in I} a_{\iota}^{p / q}\left\|F_{\iota}\right\|_{\phi_{\infty}}^{p}\left\|E_{\iota}\right\|_{\phi_{q}}^{p}\right]^{1 / p} \\
& \leqq\left[\sum_{\iota \in I} a_{\iota}^{p / q} c^{p} a_{\iota}^{(p / s)-p}\left\|E_{\iota}\right\|_{\phi_{p}}^{p}\right]^{1 / p} \\
& =c\left[\sum_{\iota \in I} a_{\iota}\left\|E_{\iota}\right\|_{\phi_{p}}^{p}\right]^{1 / p} \\
& =c\|E\|_{p} .
\end{aligned}
$$

Thus, $F E \in \mathscr{E}_{q}$ so that $F \in \mathscr{C}\left(\mathscr{E}_{p}, \mathscr{E}_{q}\right)$. Hence, $\mathscr{E}_{s}^{*} \subset \mathscr{M}\left(\mathscr{E}_{p}, \mathscr{E}_{q}\right)$.

On the other hand, suppose $T_{F} \notin \mathscr{E}_{s}^{*}$. Again, by (3.4), we have that for every $c>0$, there exists $\iota \in I$ such that $\left\|F_{c}\right\|_{\phi_{\infty}}>c \alpha_{t}^{(1 / s)-1}$. Or, in particular, for each $n \in\{1,2, \cdots\}$, let $\iota_{n}$ be such that $\iota_{n} \neq \iota_{m}$ for $n \neq m$ and

$$
\left\|F_{\iota_{n}}\right\|_{\phi_{\infty}}>n^{k} a_{t_{n}}^{(1 / s)-1}
$$

where $k$ is a real number satisfying $k \geqq 2 / p-1 / q$; that is, $1 \geqq$ $q(2 / p-k)$. For each $n \in\{1,2, \cdots\}$, let $B_{\iota_{n}} \in \mathscr{B}\left(H_{\iota_{n}}\right)$ be such that $\left\|B_{\iota_{n}}\right\|_{\phi_{p}}=1$ and $\left\|F_{\iota_{n}} B_{\iota_{n}}\right\|_{\phi_{q}}=\left\|F_{\iota_{n}}\right\|_{\phi_{\infty}}$ as in (3.1). Let $b_{n}=\left(a_{\iota_{n}} n^{2}\right)^{-1 / p}$ and define $E_{\iota}=b_{n} B_{\iota_{n}}$ if $\iota=\iota_{n}$ and $E_{\iota}=0$ otherwise. Let $E=\left(E_{\iota}\right)_{\iota \in I}$. Then

$$
\|E\|_{p}^{p}=\sum_{n=1}^{\infty} a_{\iota_{n}}\left\|b_{n} B_{\iota_{n}}\right\|_{\phi_{p}}^{p}
$$




$$
=\sum_{n=1}^{\infty} a_{\iota_{n}}\left(a_{\iota_{n}} n^{2}\right)^{-1}=\sum_{n=1}^{\infty} 1 / n^{2}<\infty
$$

so that $E \in \mathscr{E}_{p}$. However,

$$
\begin{aligned}
\|F E\|_{q}^{q} & =\left.\sum_{n=1}^{\infty} a_{\iota_{n}}\left\|F_{\iota_{n}} b_{n} B_{\iota_{n}}\right\|\right|_{\dot{o}_{q}} ^{q} \\
& =\sum_{n=1}^{\infty} a_{\iota_{n}}\left(a_{\iota_{n}} n^{2}\right)^{-q / p}\left\|F_{\iota_{n}}\right\|_{\dot{\rho}_{\infty}}^{q} \\
& \geqq \sum_{n=1}^{\infty} a_{\iota_{n}}\left(a_{\iota_{n}} n^{2}\right)^{-q / p} n^{q / v} a_{\iota_{n}}^{c / s-q} \\
& =\sum_{n=1}^{\infty} n^{q(k-2 / p)} \geqq \sum_{n=1}^{\infty} 1 / n=\infty .
\end{aligned}
$$

Thus, $F E \notin \mathscr{E}_{q}$ so that $F \in \mathscr{L}\left(\mathscr{E}_{p}, \mathscr{E}_{q}\right)$. We have, therefore, that $\mathscr{C}\left(\mathscr{E}_{p}, \mathscr{E}_{q}\right) \subset \mathscr{E}_{*}^{*}$ and $(4,2)$ is verified.

Part IV. We verify entries $(3,3),(3,4),(4,3)$ and $(4,4)$ by showing that for $0<p<\infty$,

$$
\mathscr{E}_{s}^{*}=\mathscr{L}\left(\mathscr{E}_{p}, \mathscr{E}_{0}\right)=\mathscr{L}\left(\mathscr{E}_{p}, \mathscr{E}_{\infty}\right) \text { where } s=\frac{p}{1+p} .
$$

Proof. Let $T_{F} \in \mathscr{E}_{s}^{*}$. We will first show that $F \in \mathscr{l l}\left(\mathscr{E}_{p}, \mathscr{E}_{0}\right)$. By (3.4), there exists a constant $c>0$ such that $\left\|F_{c}\right\|_{\phi_{\infty}} \leqq c a_{t}^{1 / s-1}=$ $c a_{\iota}^{1 / p}$ for all $\iota \in I$. Let $E \in \mathscr{E}_{p}$ so that $\sum_{\iota \in I} a_{\iota}\left\|E_{\iota}\right\|_{\phi_{p}}^{p}<\infty$. Then, for $\varepsilon>0, a_{\imath}\left\|E_{\iota}\right\|_{\phi}^{p} \leqq(\varepsilon / c)^{p}$ for all except finitely many $\iota \in I$. Thus,

$$
\begin{aligned}
& \left\|F_{\imath} E_{c}\right\|_{\dot{\phi}_{\infty}} \leqq\left\|F_{c}\right\|_{\dot{\phi}_{\infty}}\left\|E_{c}\right\|_{\varphi_{\infty}} \leqq\left\|F_{c}\right\|_{\dot{\zeta}_{\infty}}\left\|E_{c}\right\|_{\dot{\rho}_{p}} \\
& \leqq c a_{\iota}^{1 / p}\left\|E_{\iota}\right\|_{\phi_{p}} \leqq c \cdot \frac{\varepsilon}{c}=\varepsilon
\end{aligned}
$$

for all except finitely many $\iota \in I$. Hence, $F E \in \mathscr{E}_{0}$ so that $F \in \mathscr{M}\left(\mathscr{E}_{p}\right.$, $\left.\mathscr{E}_{0}\right)$. Clearly $\mathscr{C}\left(\mathscr{E}_{p}, \mathscr{E}_{0}\right) \subset \mathscr{C}\left(\mathscr{E}_{p}, \mathscr{E}_{\infty}\right)$ so that it remains only to show that $\mathscr{A}\left(\mathscr{E}_{p}, \mathscr{E}_{\infty}\right) \subset \mathscr{E}_{*}^{*}$.

Suppose $T_{F} \notin \mathscr{E}_{s}^{*}$. Then by (3.4), for each $n \in\{1,2, \cdots\}$, we can choose distinct $\iota_{n} \in I$ with the property that $\left\|F_{\iota_{n}}\right\|_{\dot{\rho}_{\infty}}>n^{2 / p+1} a_{\iota_{n}}^{-1 / p}$. As in (3.1), for each $\iota \in I$, let $B_{\iota} \in \mathscr{B}\left(H_{\iota}\right)$ be such that $\left\|B_{\iota}\right\|_{\phi_{p}}=1$ and $\left\|F_{\imath}\right\|_{\dot{\phi}_{\infty}}=\left\|F_{\imath} B_{\iota}\right\|_{\dot{\phi}_{\infty}}$. For each $n \in\{1,2, \cdots\}$ let $b_{n}=\left(a_{\iota_{n}} n^{2}\right)^{-1 / p}$ and let $E=\left(E_{\iota}\right)_{\iota \in I}$ where $E_{\iota}=b_{n} B_{\iota_{n}}$ if $\iota=\iota_{n}$ and $E_{\iota}=0$ otherwise. An in Part III, it is clear that $E \in \mathscr{E}_{p}$. However, $\left\|F_{\iota_{n}} E_{\iota_{n}}\right\|_{\dot{\rho}_{\infty}}=\left\|F_{\iota_{n}} b_{n} B_{\iota_{n}}\right\|_{\phi_{\infty}}=$ $b_{n}\left\|F_{c_{n}}\right\|_{\phi_{\infty}}>n$ for $n \in\{1,2, \cdots\}$. Thus, $\|F E\|_{\infty}$ is not finite so that $F E \in \mathscr{E}_{\infty}$. Hence, $F \notin \mathscr{L}\left(\mathscr{E}_{p}, \mathscr{E}_{\infty}\right)$ and so $\mathscr{M}\left(\mathscr{E}_{p}, \mathscr{E}_{\infty}\right) \subset \mathscr{E}_{s}^{*}$.

Part V. If $0<p<q<\infty$ and $1 / p-1 / q=1 / r$, then $\mathscr{l}\left(\mathscr{E}_{q}\right.$, $\left.\mathscr{E}_{p}\right)=\mathscr{E}_{r}$. 
Proof. This result is proved for $1 \leqq p<q<\infty$ in [3, 35.4, Part VI]. That proof does not carry over to our wider range for $p, q$ and $r$, however.

The inclusion $\mathscr{E}_{r} \subset \mathscr{L}\left(\mathscr{E}_{q}, \mathscr{E}_{p}\right)$ follows immediately from (4.1). To see the opposite inclusion, suppose that $E=\left(E_{\iota}\right)_{\ell \in I}$ is in $\mathscr{E}(I)$ but not in $\mathscr{E}_{r}$. We will show that $E \notin \mathscr{M}\left(\mathscr{E}_{q}, \mathscr{E}_{p}\right)$.

Let $\gamma_{\iota}=a_{t}^{1 / r}\left\|E_{t}\right\|_{\phi_{r}}$. Since $E \notin \mathscr{C}_{r},\left\{\gamma_{t}\right\}$ does not belong to $\ell_{r}(I)$. However, since $\ell_{r}=\mathscr{A}\left(\ell_{q}, \ell_{p}\right)$, there exists $\left\{\beta_{c}\right\} \in \ell_{q}$ such that $\left\{\gamma_{c} \beta_{\ell}\right\} \notin$ $\iota_{p}$. We may, and will, choose $\beta_{\iota}$ so that $\beta_{\iota} \geqq 0$ for all $\iota \in I$. Using [6, Th. 2.3] choose $F_{\iota}$ so that $\left\|E_{\iota} F_{\iota}\right\|_{\phi_{p}}=\left\|E_{\iota}\right\|_{\phi_{r}}\left\|F_{\iota}\right\|_{\phi_{q}}$ for each $\iota \in I$ and such that $E_{\imath} \neq 0$ if and only if $F_{\iota} \neq 0$. [For example, let $F_{\iota}=$ $\left|E_{\iota}\right|^{r / q}$. That the above equality holds in this case may be seen directly using conditions for equality in Hölder's inequality for $\iota_{p}$.]

For our convenience below let $\Phi=\left\{c \in I: \gamma_{\iota} \neq 0\right\}$. Note also that $\Phi=\left\{\iota \in I: E_{\iota} \neq 0\right\}$. For $\iota \in \Phi$, let $c_{\iota}=\beta_{\iota} a_{\iota}^{-1 / q}\left\|F_{\iota}\right\|_{\phi_{q}}^{-1}$, otherwise $c_{\iota}=0$. For all $\iota \in I$, let $F_{\iota}^{\prime}=c_{\iota} F_{\iota}$ and let $F^{\prime}=\left(F_{\iota}^{\prime}\right)_{\iota \in I}$. Then

$$
\left\|F^{\prime}\right\|_{q}^{q}=\sum_{\iota \in I} a_{\iota}\left\|F_{\iota}^{\prime}\right\|_{\phi_{q}}^{q}=\sum_{\iota \in \Phi} a_{\iota} \beta_{\iota}^{q} a_{\iota}^{-1}\left\|F_{\iota}\right\|_{\phi_{q}^{q}}^{q}\left\|F_{\iota}\right\|_{\phi_{q}^{q}}^{q}=\sum_{\iota \in \Phi} \beta_{\iota}^{q} \leqq \sum_{\iota \in I} \beta_{\iota}^{q}<\infty
$$

since $\left\{\beta_{\ell}\right\} \in \ell_{q}$. Thus, $F^{\prime} \in \mathscr{E}_{q}$. However,

$$
\begin{aligned}
\left\|E F^{\prime}\right\|_{p}^{p} & =\sum_{c \in I} a_{\iota}\left\|E_{\iota} F_{\iota}^{\prime}\right\|_{\phi_{p}}^{p} \\
& =\sum_{\iota \in I} a_{\iota} c_{\iota}^{p}\left\|E_{\iota} F_{\iota}\right\|_{\phi_{p}}^{p} \\
& =\sum_{\iota \in \Phi} a_{\iota} \beta_{\iota}^{p} a_{\iota}^{-p / q}\left\|F_{\iota}\right\|_{\phi_{q}}^{-p}\left\|! E_{\iota}\right\|_{\phi_{r}}^{p}\left\|F_{\iota}^{\prime}\right\|_{\phi_{q}}^{p} \\
& =\sum_{\iota \in \Phi} a_{\iota}^{1-p / q} \beta_{\iota}^{p}\left\|E_{\iota}\right\|_{\phi_{r}}^{p} \\
& =\sum_{\iota \in \Phi}\left(a_{\iota}^{1 / r}\left\|E_{\iota}\right\|_{\phi_{r}}\right)^{p} \beta_{\iota}^{p} \\
& =\sum_{\iota \in \Phi}\left(\gamma_{\iota} \beta_{\iota}\right)^{p}=\sum_{\iota \in I}\left(\gamma_{\iota} \beta_{\iota}\right)^{p}=\infty
\end{aligned}
$$

since $\left\{\gamma_{c} \beta_{\imath}\right\} \notin \ell_{p}$. Hence $E \notin \mathscr{C}\left(\mathscr{E}_{q}, \mathscr{E}_{p}\right)$ and $(3,1)$ is verified.

Part VI. $\mathscr{L}\left(\mathscr{E}_{0}, \mathscr{E}_{0}\right)=\mathscr{M}\left(\mathscr{E}_{0}, \mathscr{E}_{\infty}\right)=\mathscr{E}_{\infty}$

Proof. The proof in [3, 35.4, Part III] can be adapted to our somewhat more general setting. However, an easy direct proof will be given.

Since $\mathscr{E}_{0}$ is an ideal of $\mathscr{E}_{\infty}$, we have $\mathscr{E}_{\infty} \subset \mathscr{L}\left(\mathscr{E}_{0}, \mathscr{E}_{0}\right)$. Also, clearly, $\mathscr{M}\left(\mathscr{E}_{0}, \mathscr{E}_{0}\right) \subset \mathscr{M}\left(\mathscr{E}_{0}, \mathscr{E}_{\infty}\right)$. Thus we need to show only that $\mathscr{C}\left(\mathscr{E}_{0}, \mathscr{E}_{\infty}\right) \subset \mathscr{E}_{\infty}$. Consider any $E$ in $\mathscr{E}(I)$ that is not in $\mathscr{E}_{\infty}$. Then for each $n \in\{1,2, \cdots\}$, let $\iota_{n}$ be such that $\iota_{n} \neq \iota_{m}$ for $n \neq m$ and $\left\|E_{\iota_{n}}\right\|_{\phi_{\infty}}>n^{2}$. Let $F=\left(F_{\iota}\right)_{\iota \in I}$ where $F_{\iota}=(1 / n) I_{d_{\iota_{n}}}$ for $\iota=\iota_{n}$ and $F_{\iota}=$ 
0 otherwise. Then we have $F \in \mathscr{E}_{0}$ and $E F \notin \mathscr{E}_{\infty}$, so that $E \notin \mathscr{C}\left(\mathscr{E}_{0}\right.$, $\left.\mathscr{E}_{\infty}\right)$. Hence, entries $(2,3)$ and $(2,4)$ are verified.

Part VII. It remains only to verify $(1,3)$ by showing that $\mathscr{H}\left(\mathscr{E}_{\infty}, \mathscr{E}_{0}\right)=\mathscr{E}_{0}$.

Proof. The proof is easy. Namely, $\mathscr{E}_{0} \subset \mathscr{l}\left(\mathscr{E}_{\infty}, \mathscr{E}_{0}\right)$ since $\mathscr{E}_{0}$ is an ideal in $\mathscr{E}_{\infty}$. Finally, suppose $E \notin \mathscr{E}_{0}$. If $F_{\iota}=I_{d_{\iota}}$, then $F \in \mathscr{E}_{\infty}$ but $E F \notin \mathscr{E}_{0}$ so that $E \notin \mathscr{M}\left(\mathscr{E}_{\infty}, \mathscr{E}_{0}\right)$.

\section{BIBLIOGRAPHY}

1. N. Dunford and J. Schwartz, Linear operators, part II: spectral theory, Interscience, New York, 1963.

2. I. C. Gohberg and M. G. Krein, Introduction to the theory of linear non-selfadjoint operators in Hilbert space, Izdat. "Nauka," Moscow, 1965; also Transl. Math. Monographs, Vol. 18, Amer. Math. Soc., Providence, 1969.

3. E. Hewitt and K. A. Ross, Abstract harmonic analysis, Vol. II, Springer-Verlag, Heidelberg and New York, 1970.

4. E. Hewitt and K. Stromberg, Real and abstract analysis, Springer-Verlag, New York, 1965.

5. R. A. Kunze, $L_{p}$ Fourier transforms on locally compact unimodular groups, Trans. Amer. Math. Soc. 89 (1958), 519-540.

6. C. A. McCarthy, $c_{p}$, Israel J. Math. 5 (1967), 249-271.

Received January 21, 1970, These results were obtained in the author's 1969 doctoral dissertation written at the University of Oregon under the direction of Kenneth A. Ross.

The UNIVersity of NeW Hampshire 


\section{PACIFIC JOURNAL OF MATHEMATICS}

\section{EDITORS}

H. SAmelson

Stanford University

Stanford, California 94305

RICHARd PieRCe

University of Washington

Seattle, Washington 98105

\author{
J. DugundJI \\ Department of Mathematics \\ University of Southern California \\ Los Angeles, California 90007 \\ RICHARD ARENS \\ University of California \\ Los Angeles, California 90024
}

ASSOCIATE EDITORS
E. F. BECKENBACH
B. H. NeumanN
F. WOLF
K. YosHIDA

\section{SUPPORTING INSTITUTIONS}

UNIVERSITY OF BRITISH COLUMBIA

CALIFORNIA INSTITUTE OF TECHNOLOGY

UNIVERSITY OF CALIFORNIA

MONTANA STATE UNIVERSITY

UNIVERSITY OF NEVADA

NEW MEXICO STATE UNIVERSITY

OREGON STATE UNIVERSITY

UNIVERSITY OF OREGON

OSAKA UNIVERSITY

UNIVERSITY OF SOUTHERN CALIFORNIA
STANFORD UNIVERSITY

UNIVERSITY OF TOKYO

UNIVERSITY OF UTAH

WASHINGTON STATE UNIVERSITY

UNIVERSITY OF WASHINGTON

AMERICAN MATHEMATICAL SOCIETY CHEVRON RESEARCH CORPORATION TRW SYSTEMS

NAVAL WEAPONS CENTER 


\section{Pacific Journal of Mathematics}

\section{Vol. 35, No. $1 \quad$ September, 1970}

B. D. Arendt and C. J. Stuth, On the structure of commutative periodic semigroups ..................................... 1

B. D. Arendt and C. J. Stuth, On partial homomorphisms of semigroups .... 7

Leonard Asimow, Extensions of continuous affine functions ............ 11

Claude Elias Billigheimer, Regular boundary problems for a five-term recurrence relation ................................... 23

Edwin Ogilvie Buchman and F. A. Valentine, A characterization of the

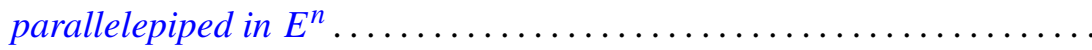
53

Victor P. Camillo, A note on commutative injective rings ............ 59

Larry Jean Cummings, Decomposable symmetric tensors ............. 65

J. E. H. Elliott, On matrices with a restricted number of diagonal values ... 79

Garth Ian Gaudry, Bad behavior and inclusion results for multipliers of type

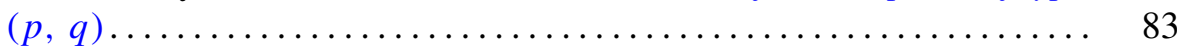

Frances F. Gulick, Derivations and actions .................. 95

Langdon Frank Harris, On subgroups of prime power index . . . . . . . . 117

Jutta Hausen, The hypo residuum of the automorphism group of an abelian

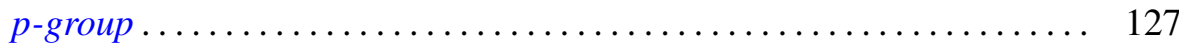

R. Hrycay, Noncontinuous multifuctions . ................... 141

A. Jeanne LaDuke, On a certain generalization of $p$ spaces ............ 155

Marion-Josephine Lim, Rank preservers of skew-symmetric matrices...... 169

John Hathway Lindsey, II, On a six dimensional projective representation of the Hall-Janko group ................................... 175

Roger McCann, Transversally perturbed planar dynamical systems . . . . . 187

Theodore Windle Palmer, Real $C^{*}$-algebras ................... 195

Don David Porter, Symplectic bordism, Stiefel-Whitney numbers, and a

Novikov resolution ....................................... 205

Tilak Raj Prabhakar, On a set of polynomials suggested by Laguerre

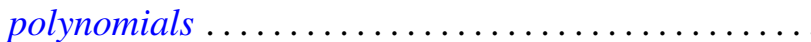

B. L. S. Prakasa Rao, Infinitely divisible characteristic functionals on locally convex topological vector spaces...................

John Robert Reay, Caratheodory theorems in convex product structures ...

Allan M. Sinclair, Eigenvalues in the boundary of the numerical range. .

David R. Stone, Torsion-free and divisible modules over matrix rings ....

William Jennings Wickless, A characterization of the nil radical of a

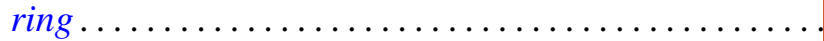

